and that he was dying from its effect. Mathews also shared in this belief, saying that from his appearance and condition he thought he had a cancerous disease and that it never occurred to him that the ulceration in the rectum could bring him to such a deplorable condition; and he further says that after the fissure was cured "in a few weeks he resumed his business at his bank, having fully recovered from his malignant(?) disease."

Doubtless the reflexes played an important part in this case as well as in some of the others herein reported. Yet it was undoubtedly the auto-infection from which this patient was suffering that caused these able men to err in their diagnosis and opinion.

I could cite other illustrations, but these cases speak for themselves and serve to show that no diseases more secretly impair and undermine the consti. tution or cause a greater degree of personal distress, or are productive of more misery and suffering or serious consequences than those occurring around the rectum and anus; and yet, notwithstanding their great importance, no class of diseases is more grievously neglected. Granting that the above were cases of autoinfection originating from disease in the rectum (and if they were not what were they?) how many more sufferers might be relieved, or even cured, by properly studying and treating pathologic conditions existing in this region?

To permit this insidious and perpetual fire of infection and disease to gradually, though slowly and surely, burn out man's existence, either by ignoring, overlooking or treating with apathy the real cause of his trouble, and constantly filling him, empirically, with drugs, when by intelligent and proper treatment this destructive flame could be so easily and completely extinguished, thereby lifting him from a pit of misery and wretchedness to that high plane of sunshine and happiness, is but little short of criminal ignorance and negligence.

103 State Street.

\section{HEREDITARY TREMOR.}

BY WM. F. WEGGE, M.D.

PROFESSOR OF MENTAL AND NERVOUS DISEASES, MILWAUKEE MEDICAL COLLEGR; ATTENDIN' NEUROLOGIST, ST. MARY'S HOSPITAI,

$$
\text { OSIIKOSH, WIS. }
$$

Owing to the small number of cases of this disease reported thus far, the following history may be of interest:

C. S., aged 3i years, single, school-teacher, has always been in rather delicate health, but has never been seriously ill. At the age of 22 years, she for the first time observed a tremor of the hands which recurred at intervals, especially when excited or exhausted from work. One year after the first appearance of the symptom, it became worse after an illness of several weeks duration. About eighteen months ago, after an exciting experience in connection with her school-work the tremor became more general, and has continued to grow worse more rapidly, ever since; so that she was finally compelled to discontinue her work on account of the annoyance caused by this distressing symptom

She states that the tremor becomes more marked in the presence of strangers, and for this reason she has avoided coming in contact with them for some time past. When alone it is very much less intense and disappears when she is at rest.

On examination the tremor is found to affect the upper extremities and the head more particularly. It is slow, rhythmic and affects speech somewhat, which is slow and strained, apparently in the effort of suppressing the tremor. Nystagmus is absent. The hand writing is clear, firm and gives no evidence of tremor. An examination of the nervous system is productive of only negative results. The patient is somewhat depressed on account of her condition and presents some neurasthenic symptoms. Aside from this the psychic functions are normal.

On further inquiry the following family history is obtained. The parents are both living. There is no history of any irregularity of habits, etc., on either side. The father has always been nervous and has been afflicted with a tremor of the hands ever since some time previous to his marriage. His mother had a tremor of the head at the age of 35 . The patient's mother's half-sister became insane at the age of 18 years, and died insane at the age of 60 years. The mother's father was periodically insane. One of the patient's sisters died during her confinement at the age of 35; was previously healthy as far as can be ascertained. Another sister, aged 25 years has had a tremor of the head for several years past. The third sister, aged 15 years, is afflicted with a tremor of the head and of the hands since childhood.

Hereditary tremor appears to be a rare disease. Brasch after a careful review of the literature on the subject has found reports of only thirty cases. $\mathrm{He}$ suggests the designation "Essential Hereditary Tremor" as the most appropriate one, since it is descriptive of the disease. The essential feature of the dis. ease is the hereditary occurrence of tremor, without any other symptoms of nervous disease.

Clinically, hereditary tremor possesses no characteristics, being subject to many individual variations. In some cases the tremor is slow, rhythmic and with slight excursions. In others it is rapid and less regular. It may be confined to the upper extremities, which appear to be most frequently affected; or it may involve these together with the head, eyes, tongue and vocal cords. The lower extremities appear to be less frequently involved. In the majority of cases it is absent when the patient is at rest, but in some cases it continues even during sleep. Excitement and excesses of every kind tend to increase it. It may develop gradually and increase progressively, or it may develop suddenly and remain stationary thereafter. The age at which the disease begins varies much. In my case it began at the age of 22 ; in the case of one of her sisters at about the same age and in the younger sister during childhood. The age at which the disease first pat in its appearance in the case of her father and in that of her paternal grandmother is indefinite, but it began rather early in life. Hereditary transmission is not always direct from parent to children, since one generation may be exempt, and the following generation inherit the disease.

More recently some authorities (Raymond and Serieux, Hamaide, Vantrin, Achard) have expressed the belief that a relationship exists between heriditary tremor and psychic degeneration.

That this relationship is a fact in all cases is certainly doubtful but in many cases one is almost forced to the conclusion that some such relationship does exist. My case is of little value in this connection. The patient's father has always been "nervous." It is evident that this may be interpreted in various ways. It certainly is very probable that the existence of a tremor would impress those about him as an evidenco of nervousness. On the other hand the patient's mu- 
ternal grandfather and aunt were insane. On first thought this fuct may look like evidence supporting the above stated belief. A more careful analysis however will lead us to the conclusion that it is not. As far as I have been able to ascertain mental or nervous diseases have not occurred on the father's side. Neither have there been any peculiarities of character or disposition. The only evidence to be found is the tremor occurring in the father and in his mother. The maternal branch of the family can not be held responsible for the tremor occurring in these two cases. Then why should it be in the case of our patient? In two cases out of the three reported by Brasch no evidence of psychic degeneration could be obtained.

In the absence of reports of autopsies in such cases we are not informed of the pathologic changes, if any exist, upon which this peculiar disease is based.

The prognosis has been unfavorable with few exceptions. Haebler has reported a case in which recovery occurred after several applications of the faradic current for the relief of an intercurrent radial paralysis. It is doubtful if other cases that have been reported recovered were true cases of hereditary tremor. In my case the neurasthenic symptoms have disappeared under treatment but the tremor continues, though less severe.

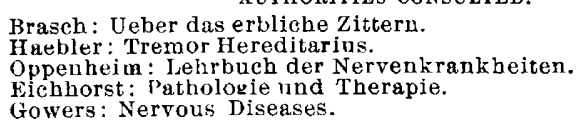

( Therapie.

\section{NITRATE OF SILVER IN DISEASES OF THE SKIN.}

Read before the Society for Medical Progress of the West Side German Dispensary, New York City, Jan. 9, 1897

BY R. ABRAHAMS, M.D.

DISTRICT PHYSICIAN TO MT. SINAI HOSPITAL, NEW YORK.

At a time when the markets are flooded with new remedies; each one backed by innumerable clinical observations, it would indeed seem reckless presumption to speak about one laden with age. But, while in the wide range of applicability the drug for which I propose to take up the cudgels this erening falls far below many of the recent ones, yet in effectiveness, circumstances and conditions remaining the same, it outdoes all the latest rivals and arrivals.

My object here is to speak about nitrate of silver, its value in the local treatment of diseases of the skin.

The physiologic action of nitrate of silver as applied locally, depends upon the strength of the salt employed. It may be caustic, escharotic, astringent and alterative, each condition resulting from the use of the mitigated, pure, saturated or very weak solution. A quality of silver nitrate passed unmentioned by writers on materia medica, but one which is probably recognized by all physicians, is this, that the salt of silver, even in its weakest state of solution, is a good antiseptic. There is little doubt but that its excellent effects in gonorrhea, ophthalmia neonatorum, etc., are due to its antimicrobic properties.

Wart.-Every variety of wart is amenable to nitrate of silver. Silver caustic has been used successfully in the removal and destruction of these papillary excrescences from time immemorial. The location of the verruca is no contraindication to the use of silver, but the size often is; for example in a case of condylomata acuminata, or venereal warts, in which the luxurious growth of the papillary buds covers a large surface, the use of silver, by reason of pain and hem- orrhage, would be impracticable as well as objectionable. The technique in all cases is simple: Work the silver stick down to the bottom of the wart, make a few sweeps around, and the papillomata, by virtue of the chemic and mechanical action of the hard ${ }^{1}$ lunar caustic, will be destroyed. Or, scrape off the lesion with spoon or finger nail and then apply the silver stick-here the salt acts as a caustic in destroying that which the spoon or nail failed to get away, and as a styptic in stopping the bleeding.

Corn.-I venture to recommend nitrate of silver in that form of epidermal callosity commonly called corn. Soak the corn in hot soapy water, then shave down the horny layers and then apply a 30 per cent. solution of silver nitrate. If personal experience is the best form of clinical evidence, then I could conscientiously testify to the effectiveness of this mode of treatment. The corn will never, or hardly ever, suffer resurrection after the silver has been applied to it.

Lupus Vulgaris.-Certain forms of lupus vulgaris are eminently adapted to the local application of nitrate of silver. The first indication is found in the small lupus papules which characterize the commencement of the destructive disease. It is evident that by effectively cauterizing the primary lesions the disease will be prevented from taking root. The second indication is suggested by the lupus nodules which are formed by the coalescence of the initial papules. Lupus in both these forms can be radically cured by and through the thorough application of silver caustic. The third indication is the serpiginous form of lupus. Here silver is used as a means only to stop the downward march of destruction. Kaposi" thus speaks of silver in the treatment of lupus vulgaris: "Apart from mechanical treatment, the use of caustic is important. The most practicable is solid nitrate of silver. It has sufficient resistance to penetrate the individual lupus nodules, thus uniting mechanical and caustic action. It also possesses the advantage that it does not enter healthy tissues. Large nodules of lupus tumidus, and particularly superficial infiltrations, may be burned out as thoroughly as with the sharp spoon. Since the solid stick not only destroys the vessels of the border and base mechanically, but also causes thrombosis, the cauterization furnishes all the requirements for effecting a cure." In the face of this eminently authoritative statement, it is hard to see why some writers of distinction fail to include this agent in the local therapeutics of lupus.

Epithelioma.-Lunar caustic finds a fitting place in suitable cases of epithelioma of the skin and mucous membranes. Generally speaking, the method of applying the caustic in cutaneous cancers is the same as in lupus or in the other growths above mentioned, but the indications are fewer. As in lupus, when the cancerous nodule or ulcer is small, nitrate of silver is an effective and curative remedy. It is also indicated in inoperable cases, in recurrent nodules and serpiginous forms of epithelioma.

The employment of the silver stick in various nonspecific ulcers on the legs or other parts of the body, applied with the object to remove spurious granulation tissue, or to freshen up indurated edges, this use of silver is so universal that it needs mention only.

1 The author, in every instance mentioned in this paper, refers to that form of lunar caustic the composition of which is: Oue part silver and three $p$ arts nitrate of potash.

2 Kaposi : Pathology and Treatment of Diseases of the Skin. American translation, page 555. 1895. Wood \& Co. 